\title{
AVALIAÇÃO DA ESCARIFICAÇÃO QUÍMICA, TÉRMICA E MECÂNICA EM SEMENTES DE Paspalum notatum Flugge BIÓTIPO BAGUAL
}

\author{
Evelise Ferreira da Silva ${ }^{1}$; Ana Carolina Silveira da Silva²; João Carlos Pinto Oliveira ${ }^{3}$ \\ ${ }^{1}$ Engenheira Agrônoma em Centro Universitário da Região da Campanha - URCAMP (Bagé); \\ ${ }^{2}$ Orientadora Dr ${ }^{\text {a }}$. Professora em Centro Universitário da Região da Campanha - URCAMP (Bagé); \\ ${ }^{3}$ Co-orientador Dr. Pesquisador da EMBRAPA Pecuária Sul. Apoio: Embrapa e FAPEG.
}

\begin{abstract}
RESUMO: Grama forquilha (Paspalum notatum) é uma gramínea nativa do bioma Pampa, produzindo forragem de qualidade, entretando a presença de dormência em suas sementes dificulta o uso em pastagens cultivadas. A escarificação de sementes é uma alternativa para a superação de dormência, buscando-se promover a melhora de um lote. Assim objetivou-se avaliar a eficiência de diferentes tipos de escarificações em sementes de $P$. notatum biótipo Bagual. Empregaram-se escarificações térmicas embebendo sementes em água a $60{ }^{\circ} \mathrm{C}$ por cinco minutos e sementes colocadas em estufa a $60 \stackrel{\circ}{\circ}$ por cinco minutos. Escarificação mecânica com lixa e escarificação química com imersão em ácido sulfúrico por diferentes tempos. Analisaram-se percentual de germinação, peso de mil sementes, umidade, emergência a campo e altura de plantas. A umidade das sementes variou de 7 a $17 \%$, o peso de mil sementes descresceu conforme maior tempo de imersão em ácido. Obtiveram-se os maiores valores de germinação nas escarificações térmicas, mecânicas e químicas nos dois menores tempos de imersão, comparado as sementes in natura. Entretanto os maiores valores na emergência foram encontrados no tratamento químico nos dois maiores tempos de imersão, e ao final de 28 dias todos tratamentos equipararam-se em altura de plantas.
\end{abstract}

Palavras-chave: Grama-Bahia, dormência, germinação, emergência.

\section{EVALUATION OF CHEMICAL, THERMAL AND MECHANICAL SCARIFICATION IN SEEDS OF Paspalum notatum Flugge BIOTYPE BAGUAL}

ABSTRACT: Bahia grass (Paspalum notatum) is a native grass for the Pampa's biome, producing qualified provender, while the presence of dormancy in its seeds 
makes it difficult to use in cultivated pastures. The scarification of seeds is an alternative to overcome dormancy, seeking an improvement of a allotment. Thus, the effectiveness of different types of scarification in seeds of $P$. notatum bagual biotype. Thermal scarification is used by soaking seeds in water at $60{ }^{\circ} \mathrm{C}$ for five minutes and the seeds placed in an oven at $60{ }^{\circ} \mathrm{C}$ for five minutes. Mechanical scarification with sandpaper and chemical scarification with immersion in sulfuric acid for different times. The percentage of germination, weight of one thousand seeds, humidity, field emergence and plant height were analyzed. The seed humidity varied from 7 to $17 \%$, the weight of one thousand seeds decriased the longest immersion time in acid. The highest germination values were reached in the thermal, mechanical and chemistry scarification in the two minors immersion times, compared as seeds in natura. However, the highest values in the emergency were found in the chemical treatment in the two largest immersion times, and at the end of 28 days all treatments were equal in height of plants.

Keywords: Bahia-grass, dormancy, germination, emergency.

\section{INTRODUÇÃO}

A pecuária brasileira é caracterizada por ter a maior parte do seu rebanho criado a pasto, considerada uma alternativa econômica para o produtor (DIAS FILHO, 2014). Segundo o IBGE (2014) apenas 60 milhões de hectares, ou seja, cerca de $36 \%$ das pastagens utilizadas para a criação de animais são formados por pastagens naturais. Porém, desde o Censo de 1970 é possível observar que as áreas de pastagens nativas veem diminuindo constantemente, sendo substituídas por lavouras ou pastagens com espécies exóticas.

Na região sul do país ainda há predominância de pastagens nativas, compostas, majoritariamente, por espécies de ciclo estival. A diversidade campestre no RS é da ordem de 2.200 espécies, sendo que as forrageiras nativas possuem grande potencial de exploração comercial devido ao seu longo período de adaptação aos locais onde são encontradas (CORADIN et al., 2011).

O projeto "Plantas para o Futuro", uma iniciativa governamental, prioriza o uso de 26 espécies de forrageiras nativas subutilizadas com o objetivo de valorizar a agrobiodiversidade natural do país. Das espécies selecionadas para o projeto, 12 são Paspalum, evidenciando a notoriedade desse gênero (CORADIN, 2011). Conforme Steiner (2005) a composição botânica de algumas das pastagens de 
regiões do estado do Rio Grande do Sul se assemelha, em qualidade, às melhores do mundo, em parte devido à contribuição de biótipos de $P$. notatum.

O gênero Paspalum é um dos mais importantes da família das Poaceas, amplamente distribuído nas regiões tropicais e subtropicais do continente americano (BALBINOT, 2007). No Brasil se encontram cerca de $75 \%$ das espécies de Paspalum conhecidas, dentre elas a grama forquilha, ou grama dos batatais ( $P$. notatum Flugge), uma espécie rizomatosa encontrada em campos pastejados (PILLAR, et al. 2009).

Ainda se desconhece a capacidade produtiva de sementes dos ecótipos Bagual e André da Rocha de $P$. notatum, apesar de sua promissora produção de forragem, chegando a $14337 \mathrm{~kg}$.ha-1 Matéria Seca Total (MST) para o biótipo bagual (MOTTA, 2014). Um dos maiores problemas encontrados para a propagação dessa espécie é a grande incidência de dormência presente nas sementes, o que diminui sua viabilidade como forrageira cultivada (BECK, 2012).

Segundo Brasil (2009) uma semente apresenta dormência quando mesmo em condições ótimas de ambiente ela não germina, podendo ser causada por ordem fisiológica, física ou presença de agentes inibidores.

Afim de obter uma melhor germinação das sementes podem ser empregadas algumas técnicas de escarificação. Para a superação de dormência diversos autores obtiveram sucesso utilizando água ou ar aquecido, materiais abrasivos ou até substâncias ácidas ou básicas como agentes escarificantes de sementes.

Santos (2017) obteve maior germinação para sementes de $P$. notatum utilizando escarificação com ácido sulfúrico por quinze minutos. Já Suñe e Franke (2006) obtiveram sucesso utilizando tratamento térmico com água aquecida por cinco minutos e tratamento mecânico com uma superfície abrasiva a fim de promover melhora na germinação de sementes de Desmanthus depressus e Trifolium riograndense.

Tendo em vista que a propagação de uma espécie está diretamente ligada à qualidade de suas sementes este Trabalho de Conclusão de Curso objetiva o estudo de diferentes técnicas de escarificação de sementes de grama forquilha ( $P$. notatum biótipo Bagual) como alternativa para a superação de dormência. Diante do exposto, 
objetivou-se avaliar a interferência do uso do tratamento de escarificação química, térmica e mecânica de sementes de $P$. notatum biótipo bagual na germinação, conhecendo as características físicas e fisiológica das sementes.

\section{MATERIAL E MÉTODOS}

Os experimentos foram realizados na Embrapa Pecuária Sul, localizada em Bagé, com as análises físicas e fisiológicas das sementes realizadas no Laboratório de Análises de Sementes em 2018. O experimento a campo foi implantado na área experimental da mesma empresa, utilizando sementes de $P$. notatum biótipo Bagual colhidas no ano de 2017.

Tratamentos: As sementes não tratadas são o grupo de controle, denominadas "testemunhas" (T1). Para escarificação térmica via úmida (T2) foram emergidas $3 \mathrm{~g}$ de sementes em $500 \mathrm{~mL}$ de água aquecida à $60{ }^{\circ} \mathrm{C}$ mantidas em um reservatório térmico por cinco minutos, após as sementes foram filtradas e encaminhadas para secagem em temperatura ambiente por 24 horas.

Já para Via Seca (T3) colocaram-se $3 \mathrm{~g}$ de sementes em uma peneira por cinco minutos em estufa de ar forçado à $60 \stackrel{\circ}{\circ}$. Na escarificação mecânica (T4): Foram escarificadas $3 \mathrm{~g}$ de sementes utilizando uma lixa d'água para retirada parcial ou total do tegumento exterior, as sementes foram friccionadas entre lixas por cerca de cinco minutos.

Finalmente, na escarificação química utilizou-se ácido sulfúrico concentrado (98\%) imergindo no máximo $1,5 \mathrm{~g}$ de sementes no mesmo, a fim de estudar qual o melhor tratamento os tempos de imersão foram de um (T5), cinco (T6), dez (T7), quinze (T8) e vinte minutos (T9). Após transcorrido o tempo em ácido as sementes foram lavadas em água corrente, durante cinco minutos, para retirada do ácido e encaminhadas para secagem em temperatura ambiente por 24 horas.

Análise Física das Sementes: Para determinação do grau de umidade colocaram-se $0,5 \mathrm{~g}$ de sementes em cadinhos, previamente pesados, em uma estufa 
à $105^{\circ} \mathrm{C}$ durante $24 \mathrm{~h}$, em duplicata. Obteve-se o valor de umidade através da diferença entre peso seco e peso verde (Equação 1).

$$
\% \text { de Umidade }(U)=\frac{100(P-p)}{P-t} \quad(\text { Equação } 1)
$$

$\mathrm{P}=$ peso inicial, peso do recipiente e sua tampa mais o peso da semente úmida; $\mathrm{p}=$ peso final, peso do recipiente e sua tampa mais o peso da semente seca; $t=$ tara, peso do recipiente com sua tampa.

Análise Fisiológica das Sementes: Para os testes de germinação seguiramse as indicações da RAS, utilizando temperatura alternada (8 horas com $35^{\circ} \mathrm{C}$ e 16 horas com $20^{\circ} \mathrm{C}$ ) e luz alternada (8 horas de luz e 16 horas de escuro), semeandose sobre substratos de papel Germitest previamente umedecidos com água destilada, após colocando-se em caixas Gerbox e incubadora tipo BOD (Biochemical Oxygen Demand).

Aplicou-se o delineamento experimental totalmente casualizado, com quatro repetições de 100 sementes por tratamento. O tempo de duração para cada teste de germinação totalizou 28 dias, com contagens a cada 7 dias após o início do teste.

Avaliação em Campo: Devido à baixa disponibilidade de sementes, cada parcela foi composta por uma linha de um metro de comprimento, com três repetições, semeando-se 100 sementes por metro linear. O delineamento experimental foi de blocos completamente casualizados. Realizaram-se contagens do número de plantas emergidas e medidas de suas alturas aos 14 e 28 dias após a data do plantio. As medidas foram de $0,25 \mathrm{~m}$ de bordadura em cada lado da linha.

Para o delineamento estatístico utilizou-se o software Statistica, versão 7.0 (Statsoft., 2004). Os resultados de germinação e emergência foram submetidos à análise de variância (ANOVA) e as médias dos tratamentos foram comparadas pelo teste de Tukey para germinação a $5 \%$ e, para a emergência Tukey a $10 \%$ de significância. 


\section{RESULTADOS}

Análise Física das Sementes: As sementes in natura possuíam $17 \%$ de umidade, enquanto que todos os demais tratamentos obtiveram valores inferiores a esse. Uma hipótese à diminuição nos valores de umidade é que o processo de escarifcação, independente por qual via, tenha facilitado as trocas entre as sementes e o ambiente. O menor valor encontrado foi de $7 \%$ referente as sementes tratadas por ar aquecido, seguido pelo tratamento de imersão em ácido por um minuto, obtendo $10 \%$ (Tabela 1).

Tabela 1: Porcentagem média dos valores obtidos para umidade. Table 1: Mean percentage of values obtained for humidity.

\begin{tabular}{cccccccccc}
\hline Tratamento & T1 & T2 & T3 & T4 & T5 & T6 & T7 & T8 & T9 \\
\hline Umidade (\%) & 17 & 11 & 7 & 10 & 10 & 13 & 15 & 13 & 16 \\
\hline
\end{tabular}

Para o peso de mil sementes encontraram-se os valores conforme a Tabela 2, abaixo. Nota-se que quanto maior o tempo de imersão no ácido, menor o peso de mil sementes, esse fato se deve ao poder corrosivo do ácido concentrado que retirou parte do tegumento das sementes.

Tabela 2: PMS para todos tratamentos das sementes de P. notatum biótipo Bagual.

Table 2: Weight of one thousand seeds for all treatments of $P$. notatum bagual biotype seeds.

\begin{tabular}{cccccccccc}
\hline Tratamento & T1 & T2 & T3 & T4 & T5 & T6 & T7 & T8 & T9 \\
\hline Peso (g) & 2,875 & 2,459 & 2,938 & 2,796 & 2,546 & 2,258 & 2,195 & 2,186 & 1,985 \\
\hline
\end{tabular}

Análise Fisiológica das Sementes: Pode-se observar, conforme Tabela 3, que a maioria dos tratamentos de escarificação utilizados obtiveram maiores valores de germinação do que a testemunha (T1).

Tabela 3: Resultados médios do teste de germinação das sementes de Paspalum notatum biótipo Bagual. Table 3: Mean results of the germination test of the Paspalum notatum Bagual biotype

\begin{tabular}{lcccc}
\hline \multicolumn{1}{c}{ Amostra } & Germinadas (\%) & Anormais (\%) & Duras (\%) & Mortas (\%) \\
\hline T1 - Testemunha & $29 \mathrm{~b}$ & $1 \mathrm{a}$ & $3 \mathrm{bc}$ & $68 \mathrm{a}$ \\
\hline T2 - Térmica Via Úmida & $67 \mathrm{a}$ & $2 \mathrm{a}$ & $7 \mathrm{a}$ & $25 \mathrm{~b}$ \\
\hline T3 - Térmica Via Seca & $56 \mathrm{a}$ & $1 \mathrm{a}$ & $7 \mathrm{a}$ & $37 \mathrm{~b}$ \\
\hline T4 - Mecânica & $65 \mathrm{a}$ & $1 \mathrm{a}$ & $4 \mathrm{ab}$ & $30 \mathrm{~b}$ \\
\hline T5 - Química $\mathrm{H}_{2} \mathrm{SO}_{4}$ Por 1 Minuto & $62 \mathrm{a}$ & $0 \mathrm{a}$ & $3 \mathrm{bc}$ & $36 \mathrm{~b}$ \\
\hline T6 - Química $\mathrm{H}_{2} \mathrm{SO}_{4}$ Por 5 Minutos & $56 \mathrm{a}$ & $0 \mathrm{a}$ & $1 \mathrm{bc}$ & $43 \mathrm{~b}$ \\
\hline
\end{tabular}




\begin{tabular}{lllll}
\hline T7 - Química $\mathrm{H}_{2} \mathrm{SO}_{4}$ Por 10 Minutos & $23 \mathrm{~b}$ & $1 \mathrm{a}$ & $0 \mathrm{c}$ & $76 \mathrm{a}$ \\
\hline T8 - Química $\mathrm{H}_{2} \mathrm{SO}_{4}$ Por 15 Minutos & $25 \mathrm{~b}$ & $1 \mathrm{a}$ & $0 \mathrm{c}$ & $88 \mathrm{a}$ \\
\hline T9 - Química $\mathrm{H}_{2} \mathrm{SO}_{4}$ Por 20 Minutos & $34 \mathrm{~b}$ & $0 \mathrm{a}$ & $0 \mathrm{c}$ & $66 \mathrm{a}$ \\
\hline $\begin{array}{l}\text { Médias seguidas pela mesma letra na coluna não diferem estatisticamente entre si pelo Teste de Tukey a } 5 \% \text { de } \\
\text { probabilidade. }\end{array}$
\end{tabular}

Avaliação em Campo: Na primeira avaliação a campo, após 3 dias da semeadura, não houve emissão de plântulas. A segunda contagem ocorreu somente após 14 dias do início do experimento pois o mau tempo impossibilitou a entrada de pessoal na área experimental nos dias anteriores. As sementes tratadas com ácido concentrado emergiram mais rapidamente e em maior número que as demais escarificações e também do que a testemunha.

Conforme a Tabela 4 observa-se que em 14 dias as sementes in natura (T1) tinham apenas sete plantas emergidas, chegando a 13 ao final de 28 dias, enquanto que as escarificadas com ácido durante maiores tempos (T7, T8 e T9) obtiveram 23, 27 e 28 plantas, respectivamente, emergidas na contagem em 14 dias e 33 ao final da avaliação, em 28 dias. Apenas o tratamento T2, obteve menor emergência entre todos tratamentos, ao contrário do resultado obtido nas condições de laboratório.

Tabela 4: Resultados médios da avaliação da emergência das sementes de Paspalum notatum biótipo Bagual após 14 e 28 dias.

Table 4: Mean results of the evaluation of the emergence of Paspalum notatum Bagual biotype after 14 and 28 days.

\begin{tabular}{lcc}
\hline \multicolumn{1}{c}{ Amostra } & Emergência após 14 dias (\%) & Emergência após 28 dias (\%) \\
\hline T1 - Testemunha & $7 \mathrm{bc}$ & $13 \mathrm{~b}$ \\
\hline T2 - Térmica Via Úmida & $0 \mathrm{c}$ & $13 \mathrm{~b}$ \\
\hline T3 - Térmica Via Seca & $4 \mathrm{abc}$ & $21 \mathrm{ab}$ \\
\hline T4 - Mecânica & $9 \mathrm{bc}$ & $17 \mathrm{ab}$ \\
\hline T5 - Química $\mathrm{H}_{2} \mathrm{SO}_{4}$ Por 1 Minuto & $11 \mathrm{abc}$ & $19 \mathrm{ab}$ \\
\hline T6 - Química $\mathrm{H}_{2} \mathrm{SO}_{4}$ Por 5 Minutos & $23 \mathrm{ab}$ & $27 \mathrm{ab}$ \\
\hline T7 - Química $\mathrm{H}_{2} \mathrm{SO}_{4}$ Por 10 Minutos & $23 \mathrm{ab}$ & $33 \mathrm{a}$ \\
\hline T8 - Química $\mathrm{H}_{2} \mathrm{SO}_{4}$ Por 15 Minutos & $27 \mathrm{a}$ & $33 \mathrm{a}$ \\
\hline T9 - Química $\mathrm{H}_{2} \mathrm{SO}_{4}$ Por 20 Minutos & $28 \mathrm{a}$ & $33 \mathrm{a}$ \\
\hline \multicolumn{2}{c}{ Médias seguidas pela mesma letra na coluna não diferem estatisticamente entre si pelo Teste de Tukey }
\end{tabular}

a $10 \%$ de probabilidade.

As alturas de plântulas apresentaram pouca diferenciação entre os tratamentos ao decorrer do teste, como mostra a Figura 1, apenas as sementes in 
natura obtiveram em média de $4,3 \mathrm{~cm}$ de altura após 27 dias, enquanto que os demais tratamentos atingiram valores igual ou superior a $5 \mathrm{~cm}$. As sementes tratadas com ácido sulfúrico emergiram mais rapidamente, porém ao final do teste equipararam-se em comprimento aos demais tratamentos.

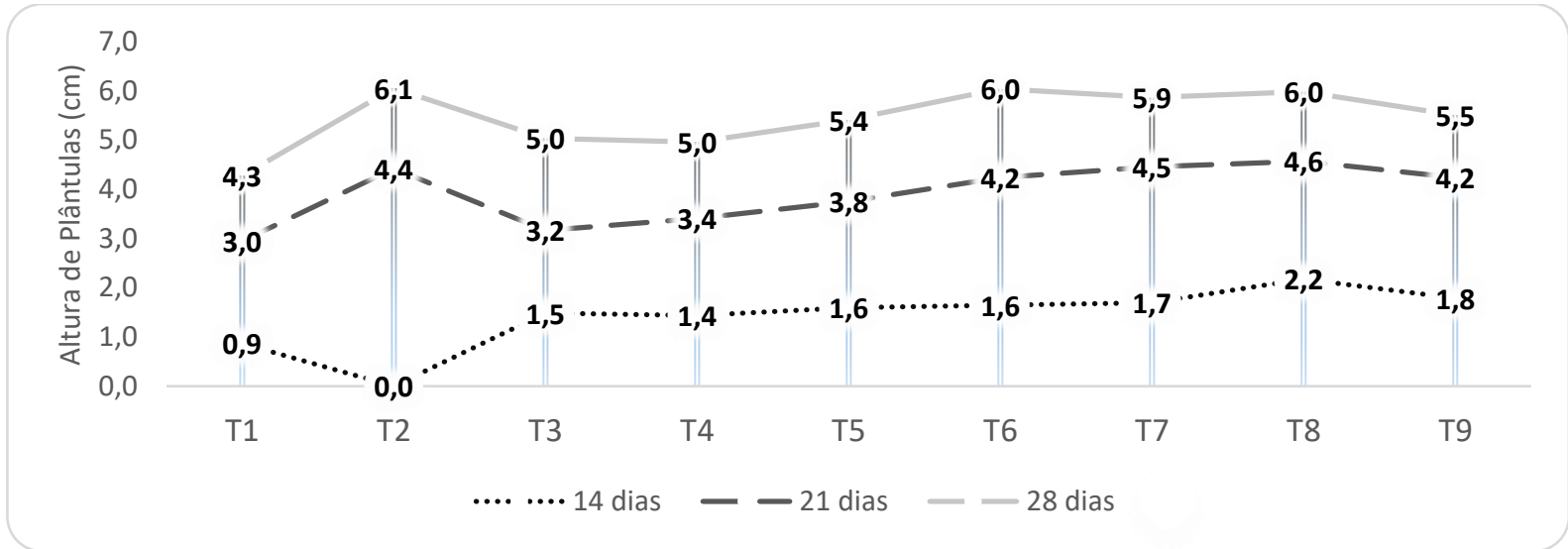

Figura 1: Resultados médios da altura de plantas de Paspalum notatum biótipo Bagual após 14, 21 e 27 dias do início do teste.

Figure 1: Mean plant height results of Paspalum notatum Bagual biotype after 14, 21 and 27 days from the beginning of the test.

\section{DISCUSSÃO}

As sementes in natura tiveram $29 \%$ de suas sementes germinadas, enquanto as escarificadas com ácido sulfúrico nos menores tempos utilizados (T5 e T6) obtiveram quase o dobro desse valor, totalizando $62 \%$ e $56 \%$ respectivamente. Suñe (2006) encontrou resultado semelhante para superação de dormência de sementes de $P$. lividum e $P$. pumilum imersas $\mathrm{em}_{2} \mathrm{SO}_{4}$ por cinco minutos.

Quando aplicado ácido sulfúrico por 15 minutos (T8) obteve-se um pequeno decréscimo na germinação, comparando-se com as sementes sem tratamento, embora não significativo estatisticamente, o resultado foi oposto ao encontrado por Santos (2017), que obteve melhora de alguns lotes de $P$. notatum estudados utilizando o mesmo procedimento de escarificação química. 
A escarificação mais eficiente foi resultante da utilização com água aquecida (T2), atingindo 67 \% de germinação, seguido da escarificação mecânica (T4) com $65 \%$, resultado semelhante obtido por Suñe (2006) em sementes de Trifolium riograndense escarificadas com água aquecida e também por Franke \& Nabinger (1996) no qual registraram melhora de alguns acessos de sementes de $P$. notatum utilizando escarificação mecânica.

\section{CONCLUSÕES}

Embora a maioria dos tratamentos para superação de dormência das sementes de $P$. notatum tenham se mostrado eficazes em laboratório é importante observar a discrepância obtida na avaliação a campo. A retirada excessiva de tegumento pela escarificação química pode levar a uma maior incidência de fungos, devido a perda da camada protetora das sementes, afetando negativamente a germinação em gerbox. Porém, em condições mais adversas a escarificação ácida pode promover maior rapidez de emergência, enquanto que sementes escarificadas por outras vias podem levar mais tempo para emergirem no solo.

\section{REFERÊNCIAS}

BALBINOT, Nair Dahmer. Variabilidade Citogenética Em Uma Coleção De Acessos De Paspalum Notatum Flügge. Universidade Federal do Rio Grande do Sul, 2007.

BECK, A. P. A. Produção De Sementes De Dois Ecótlpos De Paspalum Notatum Flügge Sob Diferentes Doses De Nitrogênio E Regimes De Corte. 2012. 140 f. Dissertação (Mestrado) - Programa de Pós-Graduação em Zootecnia, Faculdade de Agronomia, Universidade Federal do Rio Grande do Sul, Porto Alegre, 2012.

BRASIL. Ministério da Agricultura, Pecuária e Abastecimento. Regras para Análise de Sementes. Brasília: Mapa/ACS, 2009. 399p.

CORADIN, L.; SEMINSKI, A.; REIS, A. Espécies Nativas Da Flora Brasileira De Valor Econômico Atual Ou Potencial: Plantas Para O Futuro - Região Sul. Brasília: MMA, 934p., 2011.

DIAS-FILHO, M. B. Diagnóstico Das Pastagens No Brasil. Belém, PA: Embrapa Amazônia Oriental, 2014. $36 \mathrm{p}$. 
FRANKE, L. B.; NABINGER, C. - Avaliação da germinação de sementes de seis acessos de Paspalum notatum Fugge nativos do Rio Grande do Sul. Revista Brasileira de Sementes, Brasília, v 18, n.1, p102-107, 1996.

INSTITUTO BRASILEIRO DE GEOGRAFIA E ESTATÍSTICA - IBGE. Bioma do Brasil. 2006. Disponível em: <http://www.ibge.gov.br/home/presidencia/noticias/noticia_visualiza.php?id_noticia=169>

MOTTA, Eder Alexandre Minski da. Avaliação De Caracteres Agronômicos Em Híbridos Interespecíficos Do Gênero Paspalum. Tese (mestrado) - Universidade Federal do Rio Grande do Sul. Faculdade de Agronomia. Programa de Pós-Graduação em Zootecnia, Porto Alegre, BR-RS, 2014.

PILLAR, V. D. P.; MÜLLER, S. C.; CASTILHOS, Z. M. S.; JACQUES, A. V. A. Campos Sulinos - Conservação E Uso Sustentável Da Biodiversidade. Brasília: MMA, 403 p. 2009.

SANTOS, Andreza Gonçalves dos Propagação De Axonopus Parodii, Paspalum notatum E Paspalum lepton Para Cultivo De Gramados. Universidade Federal Rural de Pernambuco. Programa de Pós-Graduação em Melhoramento Genético de Plantas, 2017. Departamento de Agronomia.

STATSOFT, Inc. (2004). STATISTICA (data analysis software system), version 7 . www.statsoft.com.

STEINER, Marcelo Gomes. Caracterização Agronômica, Molecular E Morfológica De Acessos De Paspalum notatum Flugge E Paspalum guenoarum Arech Dissertação (Mestrado) - Programa de Pós-Graduação em Zootecnia, Faculdade de Agronomia. Universidade Federal do Rio Grande do Sul, Porto Alegre, 2005.

SUÑÉ, A. D.; FRANKE, L. B. SUÑÉ, A. D.; FRANKE, L. B. Metodologia De Testes De Germinação E De Vigor Para Sementes De Leguminosas E Gramíneas Nativas De Importância Para O Bioma Pampa. Tese de mestrado, Universidade Federal do Rio Grande do Sul, Faculdade de Agronomia, Porto Alegre, 2006.

SUÑÉ, A. D.; FRANKE, L. B. Superação de dormência e metodologias para testes de germinação em sementes de Trifolium riograndense Burkart e Desmanthus depressus Humb. Revista Brasileira de Sementes, 28 (3): 29-36, 2006. 